in: Petra C. Gruber (Hrsg.)(2010) Wie wir überleben! Ernährung und Energie in Zeiten des Klimawandels. Verlag Barbera Budrich pp.143-157

\title{
LOKALE BIOPRODUKTE ALS CHANCE FÜR REGIONALENTWICKLUNG?
}

Markus Schermer

Die Region hat Saison. Viele ländliche Gebiete versuchen sich in einem „Wettbewerb der Regionen“ unter einem bestimmten Thema zu positionieren, sei es im Tourismus als Urlaubs- und Erholungsdestination (z.B. Thermenregionen) oder als Standort für bestimmte Produktionszweige und Cluster (z.B. Biotechnologieregionen in Deutschland oder die "Industrial Districts“ in Teilen Norditaliens). Diese Strategie wird auch im Lebensmittelbereich angewandt. Hier werden bestimmte Leitprodukte oder auch Produktionsweisen als Ausgangspunkte für regionale Entwicklungsprozesse gewählt.

Damit gewinnt die regionale Produktherkunft an Bedeutung. Genussregionen, Bioregionen, gentechnikfreie Regionen etc. sind Beispiele und Belege dafür aus Österreich. Der Trend in diese Richtung ist aber durchaus international. Die Konzepte basieren dabei entweder auf der Bekanntheit einer Region für ein bestimmtes Produkt, das dann überregional vermarktet wird, oder auf der räumlichen Nähe zwischen bäuerlichem/r Hersteller/in und Verbraucher/in. Im ersteren Fall spricht man auch von localised Products im Gegensatz zu den local Products, die aus der gleichen Region stammen, in der sie auch vermarktet und konsumiert werden (vgl. Berard und Marchenay 2008).

Während localised products durch Labels wie geschützter Ursprung (GU) oder geschützte geographische Angabe (GGA) auch von der EU unterstützt und oft auch international vermarktet werden, basieren Lebensmittelsysteme mit local products auf Beziehungen des Vertrauens zwischen Produzent/innen und Konsument/innen, die aus der räumlichen Nähe entstehen. Ein Beispiel dafür ist die rasche Ausbreitung alternativer Lebensmittelnetzwerke, wie etwa der europaweite Boom von Biokistensystemen oder die weltweit auszumachende Entstehung neuer Allianzen zwischen Produzent/innen und Konsument/innen. Diese zeigen sich in unterschiedlichen Formen, wie CSA (Comunity Supported Agriculture) in den USA, AMAP (Associations pour le Maintien d'une Agriculture Paysanne) in Frankreich oder G.A.S (Gruppi di Acquisto Solidali) in Italien. Alternative, lokale Lebensmittelsysteme kombinieren häufig die Charakteristika von gentechnikfreier, biologischer und regionaler Herstellung zu einem gemeinsamen Produkt. Sie bleiben auch nicht bei rein wirtschaftlichen Zielsetzungen stehen, sondern bauen auf der Entstehung neuer sozialer Netzwerke auf und erheben oft den Anspruch, die Gesellschaft zum Besseren zu verändern.

Sowohl Systeme von localised Products als auch von local Products haben Auswirkungen auf die regionale Entwicklung: Beide können die regionale Wertschöpfung erhöhen. Im vorliegenden Beitrag beschränke ich mich jedoch auf local Products bzw. lokale Lebensmittelsysteme, wobei ich zunächst im folgenden Abschnitt die Potenziale der Produktion, Verarbeitung und Vermarktung von biologisch, lokal und gentechnikfrei produzierten Lebensmitteln für die Regionalentwicklung aufzeigen werde. In einem zweiten Abschnitt werden die gesellschaftlichen Ansatzpunkte für eine (Re-)Lokalisierung des Lebensmittelsystems genauer beleuchtet. Der dritte Abschnitt diskutiert die Anforderungen und Möglichkeiten der Umsetzung in Österreich und bringt ein konkretes Beispiel, wie eine regionale Wertschöpfungskette in der mehrstufigen Vermarktung aufgebaut sein kann. Abschließend wird die Frage diskutiert, ob sich eine Lokalisierung des Lebensmittelsystems langfristig gegen die Globalisierung durchsetzen kann.

\section{Potenziale lokaler Biolebensmittel für die Regionalentwicklung}

Der Britische Regionalforscher Terry Marsden (2003) unterteilt die Entwicklung ländlicher Räume seit dem zweiten Weltkrieg in drei Phasen:

- Die erste dieser drei Phasen war gekennzeichnet durch die Modernisierung der Landwirtschaft. Fordistische Produktionsprozesse ${ }^{1}$ dominierten und ländliche Regionen wurden hauptsächlich hinsichtlich ihrer Eignung für die industrielle Rohstoffproduktion und als Zulieferer für die Lebensmittelwirtschaft bewertet.

- Die zweite Phase wird als die „post-produktivistische Phase“ bezeichnet: Ländliche Räume werden nicht mehr so sehr als Produktionsstätten betrachtet, sie verändern sich zunehmend unter dem Gesichtpunkt der Erholung und des Tourismus zu Orten des Konsums.

1 Unter „Fordismus“ versteht man Massenproduktion für den Massenbedarf nach dem Beispiel der Automobilindustrie; in der Landwirtschaft gekennzeichnet durch Spezialisierung, Mechanisierung und Rationalisierung. 
- Die dritte Phase ist die der ländlichen Entwicklung, in der eine Re-Qualifikation von lokalen Ressourcen erfolgt, die zu einer Differenzierung (entgegen der Standardisierung fordistischer Tendenzen) führt. In dieser Phase sind ländliche Räume sowohl Orte der Produktion als auch des Konsums.

Lokale Lebensmittel werden in dieser dritten Phase $\mathrm{zu}$ einem wichtigen Ausgangspunkt für die Regionalentwicklung. Der Fokus auf lokale Ressourcen und die Ableitung von regionalen Entwicklungspotenzialen daraus, ist (zumindest in Österreich) nicht neu. Bereits in den späten 1970er Jahren wurden Projekte der sogenannten eigenständigen Regionalentwicklung nach diesem Muster forciert (Dax 2001). Die Erfolge der eigenständigen Regionalentwicklung bildeten unter anderem auch eine Grundlage für die gebietsbezogenen Entwicklungsansätze der Europäischen Union, die als LEADER-Programm bekannt wurden. Ein wesentliches Charakteristikum dieses Ansatzes liegt darin, dass nicht nur die wirtschaftliche Stärkung der Region, sondern auch die Stärkung eines regionalen Selbstbewusstseins und des sozialen Gefüges eine explizite Zielsetzung war und ist.

Die Verbindung regionaler Lebensmittel- und Energiekreisläufe mit gentechnikfreien Zonen und biologischer Landwirtschaft erscheint im Rahmen dieses Ansatzes von ländlicher Entwicklung nur konsequent. In Österreich sind derartige Bemühungen auch unter dem Begriff der Bioregionen bekannt geworden (Groier et al. 2008). Die Idee der Bioregion tauchte sowohl als Konzept der Regionalentwicklung wie auch der Bioentwicklung auf. Der damalige österreichische EU-Landwirtschaftskommissär Franz Fischler erwähnte auf einer Tagung zur Berggebietsentwicklung in Alpach (Tirol) bereits im Februar 2001, dass Bioregionen vor allem in der Zusammenarbeit mit dem Tourismus einen wirtschaftlichen Mehrwert bringen könnten und ermunterte vor allem extensiv wirtschaftende Berggebiete, diesen Weg zu gehen.

Groier (1998) verwies darauf, dass die Vitalität des Berggebietes in Zukunft nicht allein über Förderungen aufrecht erhalten werden könne, weshalb vermehrte Anstrengungen im Marketingbereich notwendig seien. Eine engere Bindung an die Region böte eine Möglichkeit, dem in Zukunft auch im Biobereich zu erwartenden Konkurrenzkampf zwischen Produkten aus dem Berggebiet und Gunstlagen zu entgehen. Durch die Internationalisierung des Biohandels und die damit einhergehende Annäherung in den Verarbeitungs- und Vertriebswegen von biologisch und konventionell produzierten Lebensmitteln ist dieser Konkurrenzkampf mittlerweile längst Realität geworden. Bioregionen können daher einer neuen Profilbildung der Region dienen, die den Erwartungen der Konsument/innen besser entspricht. Wesentlicher Bestandteil einer Bioregion ist die Entwicklung aktiver Strategien, um die Region frei vom Anbau gentechnisch veränderter Pflanzen zu halten. Die Ablehnung des Einsatzes von gentechnisch veränderten Organismen (GVO) stellt ein Prinzip des biologischen Landbaus dar. Mit der Debatte um die Zulassung von gentechnisch verändertem Saatgut in der EU und der Forderung nach „Koexistenz" von jenen, die dieses verwenden wollen, und anderen, die GVO-Saatgut ablehnen, wird dieses Prinzip in Frage gestellt. Die Garantie einer gentechnikfreien Bewirtschaftungsweise kann nicht auf Basis einzelbetrieblicher Strategien abgegeben werden. So kann zum Beispiel auf Grund von Pollenflug niemand garantieren, dass sein Produkt keine GVO-Rückstände aufweist, wenn der Nachbar gentechnisch verändertes Saatgut einsetzt. Dies gilt besonders für Gebiete mit einer kleinteiligen Gliederung der Besitzstruktur, wie sie im Großteil Österreichs vorherrscht. Daher sind regionale Ansätze der Abgrenzung notwendig, die schlussendlich zur Forderung nach gentechnikfreien Zonen führen.

Bioregionen oder auch gentechnikfreie Zonen können auch als Ansatzpunkte für eine alternative regionale Entwicklung verstanden werden (vgl. Schermer und Hoppichler 2004). Die Ablehnung der Gentechnologie kann zu einer bewussten Positionierung der Region mit anderen Entwicklungsleitbildern führen, sodass Regionen oder Zonen im Rahmen eines „strategischen Nischenmanagements“ (Kemp et al. 1998) als geschützte Räume dienen, in denen Gegenmodelle zu den dominierenden Entwicklungen erprobt werden können. Nach Ansicht des niederländischen Agrarsoziologen Van der Ploeg (2000) könnte die Einrichtung derartiger Nischen zu "ländlichen Distrikten" (Iacoponi et al. 1995) führen, die einen politischen und organisatorischen Rahmen für innovative Ansätze der Regionalentwicklung bilden können. Van der Ploeg hält derartige Strategien für wesentlich kosteneffektiver als viele der derzeitigen Unterstützungsprogramme im Rahmen der Ländlichen Entwicklung.

\section{Ansatzpunkte für eine (Re-)Lokalisierung des Lebensmittelsystems}

Ein wesentlicher Ansatzpunkt für die (erneute) Herausbildung lokaler und alternativer Systeme im Lebensmittelbereich liegt in den bereits beobachtbaren negativen Auswirkungen der Globalisierung auf die heimische Landwirtschaft. In den letzten Jahren hat sich gezeigt, dass die Lebensmittelpreise in zunehmendem Maße rascher und stärker schwanken, sowohl auf der Produzent/innen- wie auch auf der Konsument/innenseite. Auch wenn nur ein kleiner Teil der weltweit erzeugten 600 Millionen Tonnen Milch weltweit gehandelt wird², bestimmt die Fluktuation auf dem Weltmarkt den Erzeugerpreis im hintersten Alpental. Während der letzten 15-

2 Die OECD schätzte noch 2004, dass lediglich $8 \%$ der weltweit erzeugten Milch auch weltweit gehandelt wird, seither kann sich dieser Prozentsatz allerdings erhöht haben. 
20 Jahre bewegten sich die Preise für Milch und Milchprodukte innerhalb einer verhältnismäßig stabilen Bandbreite. Im Sommer 2007 kam es jedoch bei allen Milchprodukten zu einem außergewöhnlich starken Anstieg der Weltmarktpreise, dem erhebliche Preisrückgänge von Herbst 2007 bis Oktober 2008 folgten. Dies hatte mit mehreren Faktoren wie etwa Witterungseinflüssen in Australien und Neuseeland, Nachfrageerhöhungen in China, dem Ersatz von Futterflächen durch Agro-Energieflächen und nicht zuletzt mit Börsenspekulationen zu tun. Die verschiedenen Einflussfaktoren schaukelten sich gegenseitig auf und konnten nicht mehr durch Aufund Abbau von Lagerbeständen ausgeglichen werden. Hinzu kam, dass auf EU-Ebene strukturkonservierende Rahmenbedingungen, wie zum Beispiel die Milchkontingentierung gelockert wurden und bis 2013 gänzlich fallen sollen.

Die Bemühungen, lokale Lebensmittelsysteme wieder zu stärken, gehen sowohl von den Produzent/innen (den Bauern und Bäuerinnen) als auch von den Konsument/innen aus: Je weniger sich Bäuerinnen und Bauern wie auch ihre Vertreter/innen auf klare, langfristige, wirtschaftliche und politische Rahmenbedingungen und Regulierungen verlassen können, desto mehr setzen sie ihre Hoffnungen darauf, dass mündige Konsument/innen durch den Kauf regionaler Produkte regionale Strukturen erhalten. So wurde in Tirol im Sommer 2009 eine Imagekampagne für heimische Milchprodukte gestartet, bei der die Tiroler Jungbauernschaft/ Landjugend riesige Milchpackungen mit dem Aufdruck „1 Liter Milch $=1 \mathrm{~m}^{2}$ Kulturlandschaft“ an markanten Punkten aufstellte.

Mit dem Hinweis, dass die Konsument/innen mit ihren Kaufentscheidungen für das Überleben der bäuerlichen Landwirtschaft verantwortlich zeichnen, finden sich die Jungbäuerinnen und Jungbauern in guter Gesellschaft. Bereits vor einigen Jahren bezeichnete der deutsche Soziologe Ulrich Beck (2002) die politischen Konsument/innen als Gegenmacht der globalen Zivilgesellschaft zur Globalisierung. Formen des politischen Konsums werden in diesem Sinne auch als soziale Bewegungen aufgefasst (vgl. Goodman/De Puis 2002; Allen et al. 2003). Die Wurzeln für diese Bewegungen liegen in der Kritik an der Anonymität von Lebensmitteln und dem Wunsch nach einem nachhaltigeren Konsum. Sie sind auch Ausdruck einer zunehmenden Verunsicherung in Zeiten von Finanzkrise, Klimawandel und Lebensmittelskandalen. Nach neueren Untersuchungen scheint das Potenzial unter den Konsument/innen steigend $\mathrm{zu}$ sein. Inzwischen soll die Konsumgruppe der sogenannten LOHAs (Lifestyle of Health and Sustainability) in Deutschland bereits 30 \% der Bevölkerung umfassen (vgl. Müllner 2008). Dieser Lebensstil lässt sich durch die Schlagworte Nachhaltigkeit, Ökologie, Gesundheit - aber ebenso auch durch Genuss, Design und Individualität charakterisieren. Bio und Fairtrade seien demnach die offensichtlichsten Gewinner dieser Bewegung. Der Trendforscher Wenzel bezeichnet in einem Interview diese „moralischen Hedonisten“ als eine „Sowohl-als-auch-Klasse“, das heißt diese Gruppe von Konsument/innen will einen nachhaltigen Lebensstil ohne Konsumverzicht oder Änderung der Konsumroutinen (vgl. Hentschel 2007).

Somit zeigt sich die Zusammenführung von Regionalität und Biolandwirtschaft als eine Entwicklung, die sowohl eine Antwort auf die ökonomischen Argumente der Produzent/innen nach einer angemessenen Wertschöpfung, als auch auf die Forderung nach ethischen und sozialen Werten der Konsument/innen bietet.

\section{Wie kann eine (Re-)Lokalisierung des Lebensmittelsystems umgesetzt werden}

Die vorangegangenen Ausführungen haben aufgezeigt, dass Lokalisierung mit einem engeren Zusammenrücken von Produzent/innen und Konsument/innen verbunden ist. Die einleitend genannten Beispiele aus Frankreich, Italien und den USA beziehen sich auf direkte Beziehungen zwischen Konsument/innen(gruppen) und Produzent/innen. Dabei steht meist ein gesellschaftspolitisches Ziel im Hintergrund. Diese Formen der Zusammenarbeit erfordern eine engagierte Gruppe von Landwirt/innen und Konsument/innen, die sich häufig treffen und bereit sind eine sehr enge Bindung einzugehen. Allen diesen Formen ist gemeinsam, dass sich Konsument/innen längerfristig zum regelmäßigen Kauf von Produkten verpflichten und dafür - meist im Voraus - über eine Art Abo-System bezahlen. Damit entsteht ein Gemeinschaftsgefühl, das die klassische Trennung von Produktion und Konsumption auflöst. Konsument/innen beginnen sich mit den bäuerlichen Produktionsbedingungen auseinanderzusetzen und reden zum Teil sogar bei der Anbauplanung und der Anbauweise mit. Teilweise geht die Bindung noch weiter. Bei den AMAPs in Frankreich arbeiten Konsument/innen manchmal sogar auf den Höfen mit und bei manchen CSA-Formen in den USA werden Konsument/innen zu Miteigentümer/innen des landwirtschaftlichen Betriebs, indem sie Anteile zeichnen.

Diese eher radikalen Formen der Re-Lokalisierung erfordern ein hohes Engagement von den beteiligten Bauern und Bäuerinnen einerseits und Konsument/innen andererseits, dafür ist ihr Austausch intensiver und geht wesentlich über das Produkt hinaus. Aber auch bei den klassischen Formen der Direktvermarktung (AbHof-Verkauf, Bauernmarkt etc.) findet eine Reihe von Kommunikationsprozessen statt, die über den bloßen Warenaustausch hinausgehen und $\mathrm{zu}$ mehr gegenseitigem Verständnis zwischen Produzent/innen und Konsument/innen führen. So ist für viele Bäuerinnen und Bauern die Direktvermarktung nicht nur aus wirtschaftlichen Gründen wichtig, sondern auch auf Grund des direkten Feedbacks, das sie für ihre Produktionsleistung seitens der Konsument/innen erhalten. 
Daten der Agrarmarketing Austria (AMA) zeigen allerdings, dass die Direktvermarktung in Österreich seit 2002 stark rückläufig ist. So hat zwischen 2002 und 2006 der Einkaufswert im Ab-Hof-Verkauf um 22,5\% und auf Bauernmärkten um 21 \% abgenommen. Einen Lichtblick bilden hier Kistensysteme und Zustelldienste, die ihren Absatz um 17,4\%, erhöhen konnten (vgl. Schermer 2007). Dies gilt auch für Bioprodukte, die von Konsument/innen häufig mit direkten Absatzwegen in Verbindung gebracht werden. Allerdings zeigen Statistiken, dass im Jahr 2006 lediglich 5 \% des Bioumsatzes auf die Direktvermarktung entfielen, während der Lebensmitteleinzelhandel mit 65 \% den Löwenanteil an Bioprodukten vermarktete. Der Naturkostfachhandel ist in Österreich traditionell schwach ausgebildet und schlug sich mit lediglich $16 \%$ zu Buche. Großküchen und Gastronomie nahmen mit $6 \%$ bereits einen höheren Anteil ein als die klassische Direktvermarktung und der Exportanteil an Bioprodukten betrug $200610 \%$. (vgl. BMLFUW 2008).

Die Gründe für den Bedeutungsverlust der Direktvermarktung liegen sowohl auf Konsument/innen- als auch auf Produzent/innenseite. Wie bereits erwähnt wollen potentielle Konsument/innen, die wohl zu einem großen Teil der bereits erwähnten Gruppe der LOHAs zuzurechnen sind, zwar nachhaltig konsumieren, ihren Konsumstil aber nicht verändern. Die Wirtschaft hat längst darauf reagiert und bietet in Supermärkten und Diskontern ein breites Sortiment an Bioprodukten an. Meist unterscheiden sich diese Produkte nicht von jenem Sortiment, das auf dem Bauernmarkt angeboten wird. Fast verschwundene, alte Tomatensorten sind heute oft eher im Supermarkt als beim Bauern direkt erhältlich. Damit ist der mühsame Extraweg zum Bauernmarkt oder die Fahrt zum Hofladen nicht mehr notwendig, um mit einem guten Gewissen konsumieren zu können.

Für viele bäuerliche Produzent/innen sind die Möglichkeiten der Direktvermarktung nur begrenzt nutzbar. Insbesondere in einem Land mit einem derart hohen Anteil an kleinstrukturierten Nebenerwerbsbetrieben wie in Österreich bilden die verfügbare Arbeitszeit und die geringe Produktionsmenge kaum überwindbare Hindernisse für die Ausweitung der Ab-Hof-Vermarktung bzw. der Vermarktung auf Bauernmärkten \& Co. Zudem haben sich die Barrieren und Hürden für Neueinsteiger/innen gegenüber der Pioniergeneration vor 20 Jahren wesentlich erhöht. So werden weitaus professionellere Verarbeitungsanlagen bereits zu Beginn der Vermarktungstätigkeit vorausgesetzt, als es früher der Fall war. Zu Pionierzeiten konnte man noch experimentieren, bevor man sich völlig auf einen neuen Betriebszweig einließ und zu investieren begann. Heute wird vielmehr im Generationswechsel die Direktvermarktung häufig wieder aufgegeben, weil die Hofübernehmer/innen die Arbeitsbelastung (und teilweise Überlastung) der Elterngeneration als inakzeptabel empfinden, obwohl der Markt manchmal durchaus gegeben wäre.

Die dominierende Stellung des Lebensmitteleinzelhandels und der relative Bedeutungsverlust direkter Vermarktungswege machen es somit notwendig, nach neuen Möglichkeiten zu suchen, wie entlang indirekter Vertriebswege eine Re-Lokalisierung umgesetzt werden kann. Über die Wirkungen auf die Regionalwirtschaft hinaus soll durch diese neuen Formen die Identifikation der Produzent/innen mit ihrem Produkt erhalten bleiben und deren Wertvorstellungen bis zu den Endkonsument8innen kommuniziert werden. Hierfür ist die regionale Produktherkunft zwar eine notwendige, aber keine hinreichende Bedingung.

Häufig sind es sogar die Supermarktketten bzw. Diskonter selbst, die versuchen, über den Einsatz regionaler Produkte das Kundenvertrauen zu erhöhen. Dazu werden Eigenmarken kreiert, die vor dem Hintergrund einer bestimmten Region agieren (wie es jahrelang die Region Hohe Tauern im Fall von Ja!Natürlich war) oder das naturnahe Image verschiedener Regionen nutzen (wie im Fall der Marke Zurück zum Ursprung). In beiden Fällen bleibt aber die Marktmacht ganz klar auf der Seite des Lebensmitteleinzelhandels. Die Handelsketten können Regionen und Lieferant/innen jederzeit austauschen, wenn es wirtschaftlich opportun erscheint.

Um den eingangs beschriebenen Ansprüchen an ein regionalisiertes Lebensmittelsystem gerecht zu werden, ist jedoch eine partnerschaftliche Vorgangsweise nötig, die entlang der Wertschöpfungskette zwischen den beteiligten Partner/innen eine Kommunikation auf gleicher Augenhöhe erlaubt. Erst dies ermöglicht es, gemeinsame Ziele zu formulieren und gemeinsame Werthaltungen und Qualitätsanmutungen zu entwickeln, die eine Voraussetzung für das Entstehen langfristiger Beziehungen darstellen.

Ein Beispiel für einen derartigen Ansatz bietet die Tiroler Bio-Genossenschaft Bioalpin. Im bewussten Gegensatz zu den Handelsmarken der Supermarktketten hat Bioalpin eine Produzent/innenmarke entwickelt. Der Vorteil dieser Produzent/innenmarke liegt darin, dass die Rechte an der Marke bei den Produzent/innen liegen, in diesem Fall bei einer Bio-Genossenschaft, und nicht beim Handelspartner. Während es eine Reihe von Herstellermarken für einzelne Produktlinien gibt (so z.B. auch in der Milchwirtschaft), kommt es relativ selten vor, dass ein breites Sortiment an Lebensmitteln - teilweise direkt vom Bauern, teilweise von kleinen Verarbeitungsbetrieben - unter einer gemeinsamen Marke im Supermarkt vermarktet wird. Genau das ist das Besondere am Ansatz von Bioalpin. Diese Bio-Genossenschaft hat sich 2002 aus bäuerlichen Produzent/innen und gewerblichen Kleinverarbeiter/innen gebildet. ${ }^{3}$ Derzeit hat Bioalpin ca. 40 Mitglieder, bei denen es sich zum Teil

3 Mehr Information dazu findet sich auf der Homepage der Genossenschaft: www.bioalpin.at. 
um einzelbetriebliche Erzeuger/innen, zum Teil um gewerbliche Verarbeiter/innen handelt. Unter den bäuerlichen Produzent/innen finden sich Gemüsebauern und -bäuerinnen ebenso, wie Hühnerhalter/innen und Obsterzeuger/innen. Neben der einzigen Tiroler Bio-Metzgerei sind fast alle Bio-Kleinsennereien Tirols Mitglied. Bioalpin fungiert hier als Dachgenossenschaft bäuerlicher Initiativen. Mittlerweile umfasst die Produktpalette mehr als 80 Erzeugnisse, darunter Milch und Milchprodukte, Wurst und Fleischwaren, Eier, Obst und Gemüse, sowie Brot. Die Genossenschaft koordiniert die Erzeuger/innen, entwickelt neue Produkte und kümmert sich um Lagerhaltung und Warenwirtschaft.

Unter der Marke Bio vom Berg setzt die Genossenschaft Bioalpin ihre Produkte vornehmlich über zwei Handelspartner, MPREIS und Bäcker Ruetz, ab. Beide Firmen sind in regionalem Familienbesitz. Die Handelskette MPREIS ist der Hauptpartner. Über sie wird eine breite Produktpalette angeboten. Für Getreide wurde mit dem Bäcker Ruetz ein eigenes Programm entwickelt: Alte Landsorten werden von den Bauern der Genossenschaft Bioalpin angebaut, Ruetz verarbeitet das Getreide und vertreibt es als Biobrot unter der Marke Bio vom Berg in seinen 50 Filialen.

Der Hauptpartner MPREIS ist eine regionale Supermarktkette in Familienbesitz. Das „M“ im Firmennamen steht für den Namen der Besitzerfamilie Mölk. Das Unternehmen ist in Tirol sehr stark regional verankert und war früher nur in Tirol vertreten. In den letzten Jahren hat MPREIS allerdings auch nach Südtirol, Salzburg und Kärnten expandiert. Insgesamt verfügt das Unternehmen über 177 Filialen ${ }^{4}$ und nimmt in Tirol mit ca. 130 Geschäften eine dominierende Stellung ein. Die Marktstrategie von MPREIS basiert auf zwei Konzepten: qualitativ hochstehender Architektur ${ }^{5}$ und Regionalität. Ein wesentliches Standbein des firmeninternen Regionalitätskonzeptes ist die Marke Bio vom Berg.

Ursprünglich - etwa zur selben Zeit als die Genossenschaft Bioalpin gegründet wurde - hatte MPREIS eigentlich vor, eine eigene Biohandelsmarke zu lancieren, entschloss sich aber dann zur Zusammenarbeit mit der neugegründeten Bio-Genossenschaft. MPREIS begleitete den Aufbau der Marke, die als „Marke der Tiroler Biobauern" präsentiert wird. Gemeinsam werden neue Produkte entwickelt und am Markt eingeführt. Der zuständige Einkäufer bei MPREIS betätigte, dass sein Kontakt zu Bioalpin wesentlich enger sei, als der zu anderen Lieferanten, die ein wesentlich größeres Volumen über MPREIS vermarkten.

Regionale Integration und Vernetzung von Akteur/innen kann vertikal (entlang der Wertschöpfungskette vom Bauern über den Verarbeiter bis zum Handel) oder horizontal erfolgen. Unter horizontaler Kooperation versteht man die Zusammenarbeit von Akteur/innen auf gleicher Stufe, aber auch zwischen Bauern und Bäuerinnen und anderen regionalen Stakeholdern. Das Besondere der Vermarktungsstruktur von Bio vom Berg liegt in der Kombination von horizontalen und vertikalen Netzwerken. Dies führt zu gegenseitigen Abhängigkeiten, die sich positiv und negativ auswirken können. Positiv wirken die Abhängigkeiten symbiotisch und stabilisieren die Beziehungen. Auf der horizontalen Ebene kann die Zusammenarbeit dazu führen, dass z.B. die BioKleinsennereien über die Struktur der Genossenschaft die Möglichkeit haben, sich auf bestimmte Produktlinien zu spezialisieren, um das Sortiment von Bio vom Berg abzugleichen. Obwohl sie weitgehend unabhängig bleiben und nur einen Teil ihrer Produktion über Bioalpin absetzen, arbeiten sie zusammen und koordinieren sich in ihrer Produktpalette. Eine andere Art von Synergien entsteht in vertikaler Hinsicht zwischen den einzelnen Stufen der Wertschöpfungskette. Der gemeinsame Markenauftritt sowie die einheitliche Etikettierung und Logistik sind sowohl Voraussetzungen für die langfristige Mengen- und Preiskoordination, als auch für die gemeinsame Produktentwicklung. Diese gegenseitigen Abhängigkeiten schränken allerdings in gewisser Hinsicht auch den Handlungsspielraum der Partner ein. So wird seitens der Konsument/innen die Marke sehr stark mit MPREIS assoziiert. Es interessieren sich zwar mittlerweile auch andere Supermarktketten für Bio vom Berg, Bioalpin muss aber sehr vorsichtig agieren und kann nur in Absprache mit der Geschäftsführung von MPREIS vorgehen. Die Supermarktkette anderseits ist an die Genossenschaft gebunden, da nur Bioalpin die Produkte von Bio vom Berg anbieten kann.

Die Kooperation zwischen MPREIS und Bioalpin war bisher wirtschaftlich sehr erfolgreich (innerhalb von fünf Jahren hat sich der Umsatz versiebenfacht). Damit taucht die Frage auf, ob und unter welchen Voraussetzungen diese Art der Kooperation als Modell für andere Initiativen gelten kann. Ein wesentlicher Punkt ist das Vorliegen bzw. Entstehen gemeinsamer Werthaltungen und Qualitätsauffassungen. Dies geht über ein gemeinsames Interesse an Bioproduktion hinaus. Interviews, die im Rahmen eines EU-finanzierten Forschungsprojektes durchgeführt wurden, ergaben, dass alle an der Wertschöpfungskette Beteiligten die Qualität von Bio vom Berg Produkten relativ ähnlich beschreiben. Demnach setzt sich diese aus Eigenschaften wie traditionelle und kleinbäuerliche Produktion, regionale Herkunft etc. zusammen. In der Regionalität bzw. der regionalen Nähe findet sich ein starker gemeinsamer Nenner für die Partner. Um erfolgreich zu sein, wird also mehr als ein gemeinsames wirtschaftliches Interesse benötigt. MPREIS betont die Verbundenheit mit der Region und das

4 Siehe auch http://www.mpreis.at

5 Das Unternehmen MPREIS hat für die Architektur seiner Filialen eine Reihe von Auszeichnungen gewonnen und war 2004 sogar auf der Architektur-Biennale vertreten. 
Anliegen die regionale Wirtschaft zu stärken und die kleinstrukturierte Tiroler Landwirtschaft zu erhalten. Bioalpin betont, dass neben der biologischen Wirtschaftweise die regionale Vermarktung das Überleben der Tiroler Berglandwirtschaft sichert und unnötigen Transitverkehr verringen kann.

Die Produzent/innenmarke Bioalpin stärkt das Vertrauen der Konsument/innen in die Produkte, die Supermarktkette wiederum bietet den Produzent/innen eine flächendeckende Vermarktungsstruktur. Mit der Aussage: „Bio vom Berg liefert das Beste aus der Tiroler Berg-Biolandwirtschaft direkt zu den Konsumenten. In die MPREIS-Filiale ums Eck", wird der Geschäftsführer von Bioalpin auf der Homepage von MPREIS zitiert. ${ }^{6}$ Damit rückt sich die Genossenschaft in die Nähe einer Direktvermarktungsinitiative, die den Supermarkt als „Drop off point" benützt, wo Konsument/innen die Produkte abholen können.

Wenn es gelingt, dieses Gefühl einer „Direktvermarktung auf Distanz“ allen an der Wertschöpfungskette Beteiligten, von den bäuerlichen Mitgliedern der Genossenschaft über den Mitarbeiter/innen der Firma MPREIS bis hin zu den Konsument/innen, zu vermitteln, können die Vorteile der Direktvermarktung auch in indirekten Vermarktungswegen erhalten bleiben. Andererseits werden die Nachteile der hohen Arbeitsbelastung dadurch überwunden. Damit könnte eine Regionalisierung des Lebensmittelsystems auch unter den eingangs erwähnten strukturellen Begrenzungen effizient umgesetzt werden.

\section{Kann sich die Lokalisierung gegen die Globalisierung durchsetzten?}

Regionalisierte Lebensmittelsysteme, wie am Beispiel der Genossenschaft Bioalpin beschrieben, stellen die dominante Wachstumslogik der Globalisierung infrage. Anstelle des Wachstums einzelner Produktionsunternehmen wächst hier das Netzwerk. Neue Produkte und Produzent/innen werden teilweise aktiv gesucht und aufgebaut. Für das einzelne Produktionsunternehmen ist dann nicht mehr Wachstum, sondern die Optimierung der verschiedenen Ressourcen das Ziel. Wachstum wird häufig durch externe Prozesse induziert und als Störung empfunden. Wenn zum Beispiel in einer Kleinsennerei die Milchanlieferung steigt (weil etwa die Bauern und Bäuerinnen für denselben Gewinn intensiver wirtschaften müssen), gerät das Gleichgewicht zwischen Arbeitskraft, Verarbeitungsanlage, Lagerraum, Logistik etc. rasch durcheinander, selbst wenn ein Markt für das Produkt gegeben wäre. Bei kleinen Betriebsstrukturen lassen Veränderungen die Kosten oft sprunghaft steigen.

Die Prinzipien der Ressourcenoptimierung und der Netzwerksausdehnung anstelle von individuellem Größenwachstum erscheinen in einem bäuerlichen Denkzusammenhang logisch, da auch „die Bäume nicht in den Himmel wachsen." Wachstum bis zur Reife und die darauf folgende Vermehrung sind organische, natürliche Prozesse, die dem bäuerlichen Denken vertraut sind. Sie könnten aber auch als Ausgangspunkte und Grundprinzipien eines alternativen Wirtschaftssystems gelten.

Die dominante Wachstumsphilosophie geht hingegen immer noch von Größeneffekten aus. Damit sollen Effizienzerhöhungen erzielt werden, die zu möglichst ökonomischen Produktionsbedingungen und niedrigen Preisen für Endverbraucher/innen führen sollten. Im Lebensmittelbereich führen diese Wachstumsprozesse zunächst aber nach wie vor zu Machtkonzentrationen und Machtverschiebungen von der Primärerzeugung hin zu Verarbeitung und Handel. Zudem haben sich die staatlichen Eingriffe in das Lebensmittelsystem gewandelt. Während früher aus Gründen der Versorgungssicherheit in die Verarbeitung und Distribution von Lebensmitteln eingegriffen wurde - man erinnere sich an das österreichische Milchwirtschaftssystem vor dem EU-Beitritt, gelten die regulierenden Eingriffe heute vorwiegend der Lebensmittelsicherheit. Damit werden Verschärfungen der Hygienerichtlinien und Auflagen in der Produktion und Verarbeitung gerechtfertigt, die einer weiteren Konzentration Vorschub leisten. Die erfüllbaren Standards werden von der Industrie definiert und berücksichtigen Kleinbetriebe bzw. regionale Strukturerfordernisse nicht.

Alternative Ansätze können sich diesen Kräften letztlich kaum entziehen, wenn nicht über die bereits erwähnten „strategischen Nischen“ aktiv geschützte Bereiche geschaffen werden. Damit können Freiräume und Experimentierfelder geschaffen werden, die kreatives Potenzial für Neues freisetzen. Die Ansätze müssen nicht auf den Lebensmittelsektor beschränkt bleiben, sondern können zu alternativen Entwicklungsleitbildern für die gesamte Region werden. Alternative Wirtschaftsformen müssen allerdings solange erhalten werden, bis sie selbsttragend sind. Andernfalls bleiben sie entweder kleine marginalisierte Inseln des Widerstandes oder sie werden vom dominierenden Regime geschluckt.

Ein Beispiel dafür ist jene Entwicklung des Biomarktes, die unter dem Begriff der „Konventionalisierung“ bekannt geworden ist. Mit der Erhöhung der Nachfrage nach Bioprodukten in Supermärkten wurde das Angebot nicht nur internationalisiert, sondern verschob sich auch immer mehr hin zu höher verarbeiteten bzw. Convenience Produkten. Das Angebot auf der größten Biomesse der Welt, der Biofach in Nürnberg, unterscheidet sich heute kaum mehr von einer konventionellen Lebensmittelmesse. Auch Energy Drinks werden bereits in Bioqualität angeboten. Vom ursprünglichen Anspruch der Biobewegung, sowohl die Produktionsweisen so

6 http://www.mpreis.at/produktvielfalt/bio/bio-vom-berg/index.htm 
umzustrukturieren, dass landwirtschaftliche Betriebe im Einklang mit der Natur wirtschaften, als auch neue Formen regionalen Konsums zu fördern ist, wenig übrig geblieben. Eine Bewegung, die auszog den Markt zu verändern, wurde von den globalisierten Marktkräften transformiert.

Es erhebt sich daraus die Frage, ob derartige Prozesse zwangsläufig ablaufen müssen, ob also das dominante Regime alternative Ansätze in jedem Fall unterdrückt - entweder durch Marginalisierung oder durch Konventionalisierung. Vielleicht ist die Frage aber auch falsch gestellt. Vielleicht geht es aber in einer postmodernen Welt gar nicht mehr um ein entweder oder zwischen Globalisierung und Lokalisierung.

Wie weiter oben bereits beschrieben, propagieren Produzent/innen die Re-Lokalisierung aus wirtschaftlichen Gründen, während Konsumentinnen hauptsächlich ethische und hedonistische Motive verfolgen. Sowohl die ökonomische Argumentation der Produzentinnen wie auch die ethische Argumentation der Konsument/innen sind eine Reaktion auf Auswirkungen der Globalisierung. So gesehen erzeugt Globalisierung erst Regionalisierung. Ohne negative Erfahrungen mit globalen Warenströmen würde uns der Wert der lokalen Beziehungen nicht bewusst. Somit sind Globalisierung und Lokalisierung keine Gegensatzpaare, sie bedingen sich vielmehr gegenseitig. Robertson (1998) spricht folgerichtig von einer Glokalisierung. Ebenso scheint das derzeit beobachtbare Wachstum alternativer Ansätze, das konventionelle System nicht abzulösen, vielmehr hat es den Anschein, dass Globalisierung und Re-Lokalisierung parallel fortschreiten.

Dies deutet auf eine Teilung des Marktes in „Masse“ und „Klasse“ hin. Man muss es sich leisten können, mit politischem Konsum die Welt zu verändern; während die Masse der Konsument/innen durch ihr Kaufverhalten den Trend zur Konzentration fördert. Zum Teil dient der Konsum von ethisch korrekten, regionalen Bioprodukten auch zur Beruhigung des schlechten Gewissens jener, die sonst dem „Geiz ist geil“-Prinzip huldigen.

Andererseits greifen erwiesenermaßen Handelsketten Markttrends rasch auf. Der relative Erfolg von Bioalpin und M-Preis könnte Nachahmer bei Bauern und Handel finden. Vielleicht werden dann aus den diversen Genussregionen und Ursprungsregionen irgendwann tatsächlich Bioregionen, die in einem partnerschaftlich organisierten Produktions- und Vertriebsnetzwerk Lebensmittel aus der Region für die Region anbieten

\section{Literatur}

Allen, P. /Fitzsimmons, M./Goodman, M./Warner, K. (2003): Shifting plates in the agrifood landscape: The tectonics of alternative agrifood initiatives in California. In: Journal of Rural Studies, Vol. 19, Nr. 1, S. 61-75.

Beck, U. (2002): Macht und Gegenmacht im globalen Zeitalter. Neue weltpolitische Ökonomie. Frankfurt/M.: Suhrkamp Verlag.

Berar, L./Marcheenay, P. (2008): From Localised Products to Geographical Indications - Awareness and Action. Bourg-en-Bresse: Centre national de la recherche scientifique Alimentsc.

BMLFUW (2008): Lebensmittelbericht Österreich 2008. Wertschöpfungskette Agrarerzeugnisse. Lebensmittel und Getränke. Wien.

Dax, T. (2001): Endogenous development in Austria's mountain regions: From a source of irritation to a mainstream movement. In: Mountain Research and Development, Vol. 21, Nr. 3, S. 231-235.

Du Puis, M./Goodman, D. (2005): Should we go "home" to eat? Towards a reflexive politics in localism. In: Journal of Rural Studies, Vol. 21, Nr. 3, S. 359-371.

Groier, M./Kirchengast, Ch./Schermer, M. (Hrsg.) (2008) Auf dem Weg zur Bioregion Ergebnisse. Erfahrungen und Reflexionen aus einem Aktionsforschungsprojekt. Forschungsbericht Nr.61. Wien: BABF.

Hentschel C. (2007): Moral ist sexy. Interview mit Dr. Eike Wenzel. In: Morgenmacher (Magazin der Metro Group), $06 / 2007$.

Iacoponi, L./Brunori, G./Rovai, M. (1995): Endogenous development and the agroindustrial district. In: Van der Ploeg, JD./Van Dijk, G. (Hrsg) : Beyond modernization. The impact of endogenous rural development. Assen: Van Gorcum, S. 28-69.

Kemp, R./Schot, J./Remco, H. (1998): Regime shifts to sustainability through processes of niche formation: the approach of strategic niche management. In: Technology Analysis and Strategic Management, Vol. 10, Nr. 2.

Müllner, A. (2008): Die Lohas sind da! Wohnen zwischen Öko und Design. In: Die Presse, 21.11.2008.

Robertson, R. (1998): Glokalisierung: Homogenität und Heterogenität in Raum und Zeit. In: Beck, U. (Hrsg.): Perspektiven der Weltgesellschaft. Frankfurt am Main.

Schermer, M./Hoppichler, J. (2004): GMO and sustainable development in less favoured areas - the need for alternative paths of development. In: Journal of Cleaner Production Vol. 12, Nr. 5, S. 479-489.

Schermer, M. (2007): Das Ende der Direktvermarktung? In: Bio Austria -Fachzeitschrift für Landwirtschaft und Ökologie, 6/07, S. $32-33$.

Van der Ploeg, JD. (2000) Revitalising agriculture: farming economically as starting ground for rural development. In: Sociologia ruralis, Vol. 40, Nr. 4, S. 497-511. 